PACS 81.40.Ef

\title{
In-situ measurements of grain rotation during annealing in ultrafine grained aluminum alloy
}

\author{
V.N. Danilenko ${ }^{1 \dagger}$, D. V. Bachurin ${ }^{1,2}$, R. R. Mulyukov ${ }^{1}$ \\ †vdan@anrb.ru \\ ${ }^{1}$ Institute for Metals Superplasticity Problems, RAS, 39 Khalturin St., 450001, Ufa, Russia \\ ${ }^{2}$ Institute for Applied Materials, Karlsruhe Institute of Technology \\ Hermann-von-Helmholtz-Platz 1, 76344, Eggenstein-Leopoldshafen, Germany
}

We present the results of in-situ studies of evolution of grain boundary misorientations in ultrafine grained (UFG) aluminum alloy Al-4Cu-0.5Zr (wt\%) during annealing. The structure of the UFG alloy was obtained by severe plastic deformation method. Misorientations between the grains were measured using single reflection technique. It was found that relaxation processes during annealing are accompanied by grain rotation. Analysis of the kinetics of grain rotation in the discretedislocation approach has demonstrated a good agreement between the numerical estimates and the experimental data.

Keywords: grain boundary, in-situ annealing, single reflection technique, ultrafine grained aluminum alloy.

\section{Introduction}

High internal stresses are typical for ultrafine grained as well as nanocrystalline materials produced by severe plastic deformation method [1-3]. During deformation and annealing various relaxation processes occur in these materials such as grain boundary sliding, grain rotation, grain boundary migration etc.

Grain rotation as a possible mechanism of microstructure evolution was studied in Ref.[4], where a two-dimensional polycrystalline system was simulated under the influence of finite temperatures, applied strains and external fields. A continuum theory of grain rotation in columnar polycrystalline microstructures was developed in Ref.[5], where grain-rotation-induced grain coalescence is considered as an additional mechanism for grain growth. Molecular dynamics simulations of a circular grain embedded in a single-crystal matrix have shown that grain boundary migration and grain rotation occur simultaneously [6].

Grain rotation of individual bulk grains subjected to plastic deformation was observed in pure aluminum by means of three-dimensional X-ray diffraction [7]. In-situ observation of grain rotations was carried out for nanostructured gold thin films [8] and nanocrystalline copper [9]. Rotation of subgrains in thin films was observed during the heating of the monocrystals of $\mathrm{Fe}-3 \% \mathrm{Si}$ after the cold rolling [10] and during the heating of Al-6\% Ni alloy in the column of the high-voltage electron microscope [11], where the grain rotation was detected by loss of dislocations from low-angle grain boundaries. Grain rotation in thin, columnar, <111> textured films of gold were determined by changing of the twins orientation within the grains [12]. However, all above listed experimental works deal with qualitative characterization of grain and subgrain rotation, while quantative studies are relative poorly covered in the scientific literature.

Investigation of evolution of the grain boundary misorientations during in-situ annealing of UFG alloy $\mathrm{Al}-4 \mathrm{Cu}-0.5 \mathrm{Zr}$ has shown that relaxation of the microstructure is accompanied by grain rotation [13]. In the present paper we continue this study.

\section{Experimental materials and procedures}

The UFG structure of aluminum alloy $\mathrm{Al}-4 \mathrm{Cu}-0.5 \mathrm{Zr}$ was obtained by means of severe plastic deformation method by shearing under the high quasi-hydrostatic pressure up to the degree of deformation of $e \approx 7$. Investigation of the sample was carried out in the column of transmission electron microscope JEM-2000EX with the help of standard heating device supplied with the microscope. The foils were prepared by jet electropolishing.

Several grain boundary misorientations were measured on the selected region of the as-prepared specimen (after deformation of $e \approx 7$ ). Thereafter the sample was annealed twice during 120 seconds at a temperature of $433 \mathrm{~K}$. Observation of the structure evolution was performed after the first and the second annealing.

Grain boundary misorientations were measured using single reflection technique [14], which is based on determination of the orientation matrix $M$ setting of oneto-one correspondence between the laboratory and the crystallographic coordinates of the reference vector. The direction toward reciprocal lattice node located on the Ewald sphere, i.e. the reciprocal lattice vector $\mathbf{g}(\mathrm{hkl})$, was chosen for the direction of the reference vector. Laboratory coordinates of this vector were fixed on the electron diffraction pattern. 
The orientation matrix was found using three non-coplanar unit reference vectors. The rotation matrix $R$ superposing the crystal lattices of two neighboring grains was calculated from the known orientation matrices of two adjacent grains $M_{1}$ and $M_{2}$ :

$$
R=T \cdot M_{1} \cdot M_{2}^{-1}
$$

where $T$ is an operator of rotational symmetry of the crystal lattice. The elements of the rotation matrix $R$ were used for calculation of misorientation angle and misorientation axis.

Possibility to determine the orientation of every single grain is the feature of single reflection technique. Using the orientation matrices of the same grain before and after annealing, the misorientation matrix and then the misorientation angle can be calculated. In this case, the misorientation angle describes the rotation of the grain during annealing. The inaccuracy of the orientation measurement for each grain was less than $0.3^{\circ}$. The maximal error in determination of the grain boundary misorientation was $0.6^{\circ}$.

\section{Results and discussion}

Figure 1a and Table 1 show the grained structure of the aluminum alloy subjected to deformation with the mean grain size of $200 \mathrm{~nm}$. The grains were found to be almost free of dislocations. However, extinction contours indicating high internal stresses are seen inside the majority of the grains. Obviously, if there is no any substructure, the grain boundaries are the sources of these stresses. Two spread dark and light bands are seen in the micrographs as well as high internal stress fields. These features are typical for such grain boundaries which absorbed lattice dislocations [1,2] and an evidence of their non-equilibrium state.

After the first annealing (see Fig.1b) the mean grain size did not change noticeably, excluding the grain 1 , which has grown. The second phase particles (identified as $\mathrm{Al}_{2} \mathrm{Cu}$ ) appeared in the triple junctions. One of these second phase particles has covered the grain 2 . Lattice dislocations were observed in the grain 1 .

The emergence of the second phase particles between the grains 3 and 4 led to a disappearance of the grain boundary $3-4$. On the scheme of the investigated region (Fig.1d) the particle of the second phase is separated from the grain 4 with the dashed line. Therefore, misorientation of the boundary 3-4 has a conditional character in the sense that such boundary can exist only in the case if there are somewhere (for instance, in a depth of the foil) the regions where the grains 3 and 4 contact each other. If such regions do not exist, then this misorientation indirectly shows the change of orientation between the grains 3 and 4 .

The first annealing did not lead to appearance of banded diffraction contrast typical for equilibrium grain boundaries, but dark and light bands become more distinct (see grain 3 in Fig.1b). The latter fact testifies that the relaxation processes is accompanied by changing of the grain boundary misorientations (see Table 1).

However, we cannot exclude that the observed misorientations change may be connected with the appearance of the second

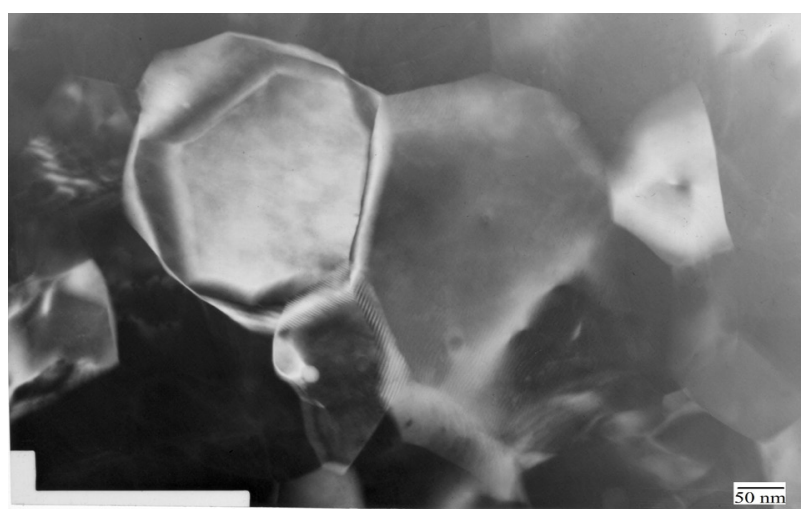

a

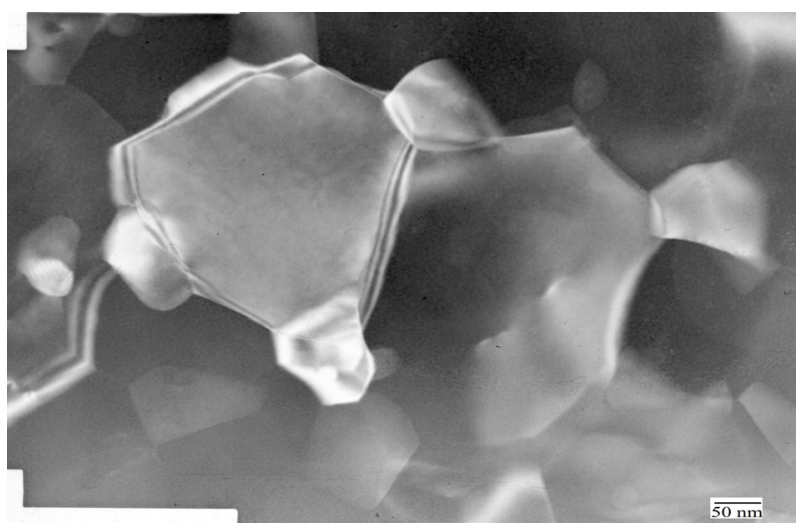

b

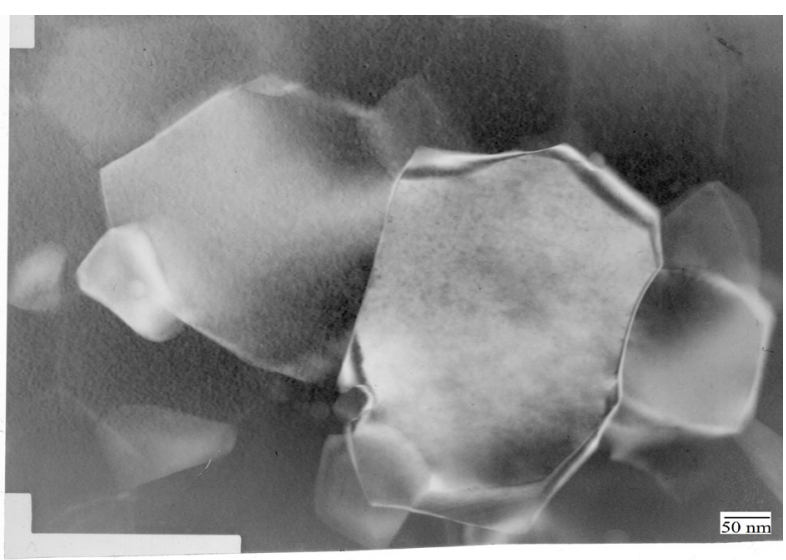

C

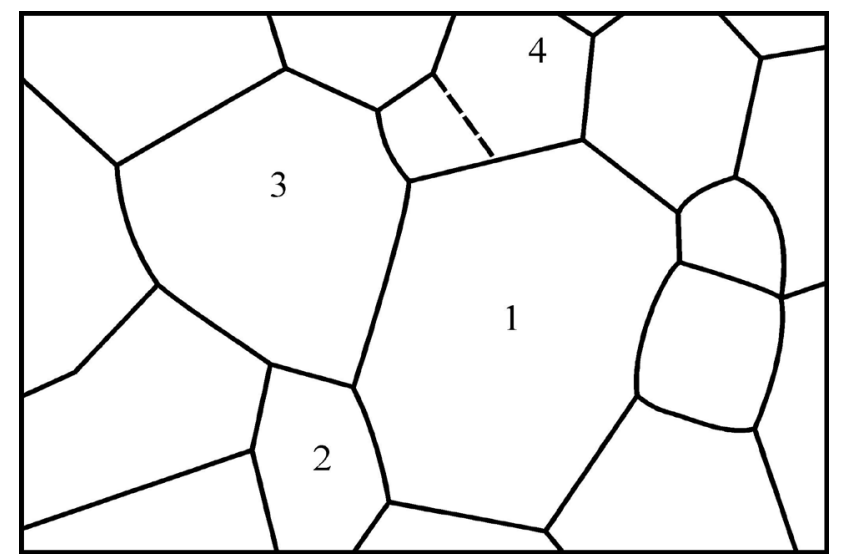

d

Fig. 1. The structure of UFG aluminum alloy: (a) after deformation of $e \approx 7$; after the first (b) and the second (c) annealing; (d) scheme of the investigated region. 
Evolution of the grain boundary misorientations in UFG aluminum alloy. The misorientation axis is shown in square brackets

\begin{tabular}{|c|c|c|c|}
\hline \multirow{2}{*}{ State of material } & \multicolumn{3}{|c|}{ Grain boundary misorientation } \\
\cline { 2 - 4 } & $1-3$ & $3-4$ & $1-4$ \\
\hline $\begin{array}{c}\text { Initial } \\
\text { (after } e \approx 7)\end{array}$ & $56.9^{\circ}$ & $28.7^{\circ}$ & $37.8^{\circ}$ \\
\hline $1^{\text {st }}$ annealing: & {$[0.7280 .6170 .300]$} & {$[0.9440 .2610 .201]$} & {$[0.8530 .4170 .320]$} \\
\hline $433 \mathrm{~K}, 120 \mathrm{sec}$. & $58.5^{\circ}$ & $31.2^{\circ}$ & $40.1^{\circ}$ \\
\hline $2^{\text {nd }}$ annealing: & {$[0.7250 .646 .0 .238]$} & {$[0.8980 .3250 .297]$} & {$[0.8190 .4760 .320]$} \\
$433 \mathrm{~K}, 120 \mathrm{sec}$. & {$[0.7170 .6170 .324]$} & {$[0.8990 .3460 .270]$} & {$[0.8180 .5040 .277]$} \\
\hline
\end{tabular}

Table 2.

Grain rotation after the first and the second annealing

\begin{tabular}{|c|c|c|c|}
\hline \multirow{2}{*}{$\begin{array}{c}\text { Change } \\
\text { of material state }\end{array}$} & grain 1 & grain 3 & grain 4 \\
\cline { 2 - 4 } & 2.3 & 2.6 & 2.4 \\
\hline Initial $-1^{\text {st }}$ annealing & 1.9 & 1.4 & 2.5 \\
\hline $1^{\text {st }}$ annealing $-2^{\text {nd }}$ annealing & & & \\
\hline
\end{tabular}

phase particles. In order to study this question the repeated annealing of the sample at the similar conditions was carried out.

After the second annealing (see Fig.1c) only a slight growth of the grains 1 and 3 was observed. Diffraction contrast on the grain boundaries became clearer, which is a sign of further decrease of internal stresses as compared with the first annealing. As seen in Table 1, the misorientation angle between the grains $1-3$ and $1-4$ has been increased. The deviation from this tendency for the boundary $3-4$ is related probably to the appearance of the second phase particle.

The changes of rotation angle between the grains during annealing are presented in Table 1 . One can see that the rotation angle is larger after the first annealing than after the second one (excluding the grain 4). It emphasizes the fact that grain rotation velocity gradually decreases with the time. The latter outcome is in accordance with the conclusions made earlier in experimental $[12,15]$ as well as in theoretical works $[16,17]$. It should be noted that the magnitude of the measured rotation angle of the grains 1, 3 and 4 several times exceeds the maximal possible error in this experiment.

Grain rotation in nanocrystalline and UFG materials has been considered theoretically in two different approaches. In the discrete-dislocation model the kinetics of the process is controlled by the forces of elastic interaction between the dislocations, while the continuum approach is based on the diffusive transport of matter from compressive regions into the tensile regions. The comparison of rotation time calculated in these two models for the boundary $1-3$ with the experimental data is shown below.

The authors [17] have investigated the kinetics of grain rotation in a bicrystal by studying the relaxation of an edge dislocation wall in the discrete-dislocation approach. The expression for the relaxation time to reach the metastable configuration as a function of misorientation angle between the two crystallites has been obtained. Using the results of [17] (see formula (20)), let us evaluate the relaxation time assuming that the grain rotation is controlled by the climb velocity of the lattice dislocations trapped by the grain boundaries. Our estimate uses the following parameters: the shear modulus $G=26 \mathrm{GPa}$, Poisson's ratio $v=0.35$, the lattice constant $a=0.405 \mathrm{~nm}$, grain boundary length $L=230 \mathrm{~nm}$, grain boundary diffusion constants $\delta D_{b}=5.0 \times 10^{-14} \cdot \exp (84000 / R T) \quad \mathrm{m}^{3} \mathrm{~s}^{-1} \quad$ [18]. The symbol $R$ denotes the gas constant and $T$ is a temperature. We obtain approximately 200 seconds. Taking into account the approximate nature of the qualitative comparison, one can conclude, that our estimates are in a good agreement with the obtained experimental data.

For comparison let us calculate the rotation time using the model proposed in Ref. [12], where the kinetics of grain rotation was considered in continuum approach. Using the same values of the parameters, we obtain $6.2 \times 10^{4}$ seconds. This is two orders of magnitude larger than our estimate in the discrete-dislocation approach.

\section{Conclusions}

In summary, we have shown that on the initial stage of in-situ annealing of UFG aluminum alloy $\mathrm{Al}-4 \% \mathrm{Cu}-0.5 \% \mathrm{Zr}$ preliminary subjected to severe plastic deformation, the grain rotation occurs, which is directly associated with the relaxation processes.

Obtained experimental data are in a good agreement with the theoretical estimates made in the discrete-dislocation approach.

\section{References}

1. N.I. Noskova, R.R. Mulyukov. Submicrocrystalline and nanocrystalline metals and alloys. Ekaterinburg: Uralskoe 
otdelenie RAS. (2003) 279 p. (in Russian)

2. R.Z. Valiev, I. V. Alexandrov. Nanostructured materials obtained by severe plastic deformation. M.: Logos. (2000) 272 p. (in Russian)

3. R.Z. Valiev, A.P. Zhilyaev, T.G. Langdon. Bulk Nanostructured Materials: Fundamentals and Applications. New Jersey. Wiley \& Sons. (2014) 440 p.

4. C.S. Nichols, R. F. Cook, D. R. Clarke, D. A. Smith. Acta Metallurgica et Materialia. 39, 1657 (1991).

5. D. Moldovan, D. Wolf, S. R. Phillpot. Acta Materialia. 49, 3521 (2001).

6. M. Upmanyu, D. J. Srolovitz, A. E. Lobkovsky, J. A. Warren, W. C. Carter. Acta Materialia. 54, 1707 (2006).

7. H.F. Poulsen, L. Margulies, S. Schmidt, G. Winther. Acta Materialia. 51, 3821 (2003).

8. M. Ke, S. A. Hackney, W.W. Milligan, E.C. Aifantis. Nanostructed Materials. 5, 689 (1995).

9. N.I. Noskova, E. G. Volkova. Physics of Metals and Metallography. 91, 100 (2001). (in Russian)
10. S.Hu. In Electron microscopy and strength of crystals. M.: Metallurgiya. (1986) 350 p. (in Russian)

11. F. J. Humphreys, H.M. Chan. Materials Science and Technology. 12, 143 (1996).

12. K. E. Harris, V. V. Singh, A.H. King. Acta Materialia. 46, 2623 (1998).

13. V.N. Danilenko, R. R. Mulyukov. Perspektivnye materialy. 12, 113 (2011). (in Russian)

14. V.V. Rybin. Large plastic deformations and fracture of metals. M.: Metallurgiya. (1986) 224 p. (in Russian)

15. H. Kuhn, G. Baero, H. Gleiter. Acta Metallurgica. 27, 959 (1979).

16. G. Martin. Physica Status Solidi. B172, 121 (1992).

17. D. V. Bachurin, A. A. Nazarov, J. Weissmueller. Acta Materialia. 60, 7064 (2012).

18. H.J. Frost, M.F. Ashby. In: Deformation - mechanism maps. Chelyabinsk. Metallurgiya. Chelyabinskoe otdelenie (1989) 328 p. (in Russian) 